\title{
Análisis de medidas no-supervisadas de calidad en clusters obtenidos por K-means y Particle Swarm Optimization
}

\author{
Andrea Villagra, Ana Guzmán, Daniel Pandolfi \\ Universidad Nacional de la Patagonia Austral, Unidad Académica Caleta Olivia, \\ Laboratorio de Tecnologías Emergentes (LabTEm) \\ Caleta Olivia, Santa Cruz, Argentina, 9011 \\ \{avillagra,aguzman,dpandolfi\}@uaco.unpa.edu.ar \\ y \\ Guillermo Leguizamón \\ Universidad Nacional de San Luis, \\ Laboratorio de Investigación y Desarrollo en Inteligencia Computacional (LIDIC) \\ San Luis, Argentina, 5700 \\ legui@unsl.edu.ar
}

\begin{abstract}
Data clustering helps in discerning the structure and simplifing the complexity of massive quantities of data. It is a common technique used in many fields, including machine learning, data mining, image analysis, and bioinformatics, in which the distribution of information can be of any size and shape. The efficiency of clustering algorithms is strongly required with very large databases and high-dimensional data types. This paper presents an evaluation study, from different perspectives, of several important unsupervised quality measures including quantization error, intra- and inter-cluster distances, obtained by the well-known K-means algorithm and a population-based metaheuristic called Particle Swarm Optimization (PSO) and a hybrid algorithm that combines the characteristics of both algorithms, called PSO+K-means. Results show that in general the PSO+K-means algorithm obtains better results in each measure and generates higher compact and separates clustering than either PSO or K-means alone.
\end{abstract}

Keywords: Clustering, K-means, Particle Swarm Optimization, Unsupervised quality measures.

\section{Resumen}

El clustering de datos ayuda a discernir la estructura y simplifica la complejidad de cantidades masivas de datos. Es una técnica común y se utiliza en diversos campos como, aprendizaje de máquina, minería de datos, reconocimiento de patrones, análisis de imágenes y bioinformática, donde la distribución de la información puede ser de cualquier tamaño y forma. La eficiencia de los algoritmos de clustering es extremadamente necesaria cuando se trabaja con enormes bases de datos y tipos de datos de grandes dimensiones. Este trabajo presenta una evaluación desde distintas perspectivas de una serie de medidas relevantes no-supervisadas de calidad como por ejemplo, cuantización del error, distancias intra- e inter- cluster, de los clusters obtenidos por el conocido algoritmo de $K$-means, una metaheurística poblacional denominada Particle Swarm Optimization (PSO) y un algoritmo híbrido, que combina las características de los dos algoritmos anteriores, denominado PSO+Kmeans. De los resultados obtenidos se observa que en general el algoritmo PSO $+K$-means obtiene mejores resultados en cada una de las medidas generando clusters más compactos y separados entre ellos que los obtenidos por los otros algoritmos.

Palabras claves: Clustering, K-means, Particle Swarm Optimization, Medidas no-supervisadas. 


\section{Introducción}

Los datos son una fuente crítica en varias organizaciones y por lo tanto la eficiencia de acceso a ellos, el compartir, extraer información y hacer uso de esa información se ha convertido en una imperiosa necesidad. Actualmente, la minería de datos conforma uno de los campos de investigación y aplicación más reconocidos para llevar a cabo dichas tareas. En términos generales, podemos decir que la minería de datos es el proceso de extraer información útil, patrones y tendencias previamente desconocidas, de grandes bases de datos. La minería de datos ha recibido un gran impulso en los últimos tiempos motivado por distintas causas: a) el desarrollo de algoritmos eficientes y robustos para el procesamiento de grandes volúmenes de datos, b) un poder computacional más barato que permite utilizar métodos computacionalmente intensivos, y c) las ventajas comerciales y científicas que han brindado este tipo de técnicas en las más diversas áreas.

Clustering es una de las tareas más utilizadas en el proceso de minería de datos para descubrir grupos e identificar interesantes distribuciones y patrones en los datos. Es un método exploratorio que ayuda a resolver problemas de clasificación. Su uso es adecuado cuando se conoce poco o nada sobre la estructura de los datos. El objetivo del clustering es organizar una muestra de casos en consideración en grupos de forma tal que el grado de asociación es alto entre los miembros del mismo grupo y bajo entre los miembros de diferentes grupos. También se llama al clustering clasificación no-supervisada, donde no hay clases predefinidas [7].

Hay dos técnicas principales de clustering "Particional" y "Jerárquica" [1], [2]. En las ultimas décadas, debido al desarrollo de la inteligencia artificial se han presentado métodos de clustering basados en otras teorías o técnicas [9], [8].

Un algoritmo de clustering muy conocido es el algoritmo de $K$-means y sus variantes. Este algoritmo es simple, directo y se basa en análisis de varianzas. La principal desventaja del algoritmo de $K$-means es que el cluster resultante es sensible a la selección inicial de los centroides y puede converger a un óptimo local. Por lo tanto, la selección inicial de los centroides dirige el proceso de $K$-means y la partición resultante está condicionada a la elección esos centroides. $K$-means realiza una búsqueda local en la vecindad de la solución inicial y va refinando la partición resultante, por esta razón se puede utilizar algún algoritmo de búsqueda global para generar los centroides iniciales. El algoritmo de cúmulo de partículas (PSO - Particle Swarm Optimization) es una técnica de optimización estocástica que puede utilizarse para encontrar una solución óptima o cercana al óptimo, ha sido aplicado en clustering de datos y de texto con muy buenos resultados [6],[10], [3]. PSO puede utilizarse para generar buenos centroides iniciales para el $K$ means. En este trabajo se utiliza un algoritmo híbrido PSO+K-means que combina la búsqueda global de PSO y la búsqueda local de $K$-means, obteniendo mejoras en las medidas de calidad analizadas.

El resto del trabajo esta organizado de la siguiente manera, en la sección 2 se describe el algoritmo de $K$-means, en la sección 3 se muestra el algoritmo de PSO, en la sección 4 se describe la aplicación de PSO a clustering y el algoritmo híbrido PSO+K-means, en la sección 5 de muestran las medidas de calidad utilizadas, en la sección 6 se detallan los experimentos y resultados obtenidos y por último en la sección 7 las conclusiones.

\section{K-means}

El algoritmo de K-means fue propuesto por MacQueen en el año 1968 [5] es simple, directo y está basado en el análisis de las varianzas. Agrupa un conjunto de datos en un número predefinido de clusters. Comienza con un conjunto aleatorio de centroides de cada uno de los clusters y continúa reasignando los datos del conjunto de datos a los centroides, basándose en la similitud entre el dato y el centroide. El proceso de reasignación no se detiene hasta que se converge al criterio de parada (por ejemplo, se alcanzó un número fijo de iteraciones o los clusters encontrados no cambian luego de cierto número de iteraciones).

El algoritmo de $K$-means puede resumirse de la siguiente manera:

1. Selecciona un conjunto aleatorio de centroides iniciales.

2. Asigna cada elemento del conjunto de datos al centroide más cercano.

3. Recalcula los centroides usando:

$$
c_{j}=\frac{1}{\left|C_{j}\right|} \sum_{\forall x \in C_{j}} z
$$

donde z representa un elemento del conjunto de datos, que pertenece al cluster $C_{j} ; c_{j}$ es un centroide y $\left|C_{j}\right|$ corresponde al número de elementos en el cluster $C_{j}$.

4. Repetir los pasos 2 y 3 hasta que se alcance la condición de parada.

Una desventaja de este algoritmo es que el resultado obtenido es dependiente de la selección inicial de los centroides de los clusters y puede converger a óptimos locales [11]. Por lo tanto, la selección de los centroides iniciales 
afecta el proceso principal de $K$-means y la partición resultante de este proceso. No obstante, si se obtienen buenos centroides iniciales con alguna técnica alternativa, $K$-means refinaría esos centroides de los clusters obteniendo mejores resultados.

\section{Particle Swarm Optimization (PSO)}

Particle Swarm Optimization (PSO) es una técnica de computación evolutiva desarrollada por Kennedy y Eberhart en 1995 [4], inspirada en el comportamiento social del vuelo de las bandadas, movimiento de los cardúmenes, entre otros sistemas sociales altamente cohesionados y que permite, simulando este modelo de comportamiento, obtener métodos eficientes para resolver problemas de optimización. En el algoritmo, los cardúmenes se representan simbólicamente como partículas. Estas partículas se consideran simples agentes que se "mueven" a través de un espacio de problema. La ubicación de una partícula en un espacio de problema multi-dimensional representa una posible solución. Cuando la partícula se mueve a una nueva ubicación, se genera una nueva solución. Esta solución se evalúa a través de una función de aptitud o "fitness" proveyendo un valor cuantitativo de la utilidad de esa solución. La velocidad y la dirección de cada partícula se ajusta de acuerdo a su propia experiencia $p_{i d}$ y a la experiencia de sus vecinos $p_{g d}$. Los valores aleatorios $r_{1}$ y $r_{2}$ y los coeficientes $c_{1}$ y $c_{2}$ controlan la influencia estocástica de las componentes cognitivas y sociales sobre la velocidad de la partícula. Para cada generación, la nueva ubicación de la partícula se calcula agregando a la posición actual de la partícula, vector $x$, la nueva velocidad, vector $v$. Matemáticamente, en un espacio de problema multi-dimensional, la $i$-ésima partícula cambia su velocidad de acuerdo a la siguiente ecuación:

$$
\begin{gathered}
v_{i d}=w * v_{i d}+c_{1} * r_{1} *\left(p_{i d}-x_{i d}\right)+c_{2} * r_{2} *\left(p_{g d}-x_{i d}\right) \\
x_{i d}=x_{i d}+v_{i d}
\end{gathered}
$$

donde $w$ es un factor de peso de inercia; $p_{i d}$ es la memoria de la partícula, es decir su mejor posición; $p_{g d}$ es la mejor posición global, es decir la ubicación de la partícula con el mejor valor de aptitud; $c_{1}$ y $c_{2}$ son los coeficientes de aceleración; $d$ corresponde a la dimensión del espacio del problema; $r_{1}$ y $r_{2}$ son valores aleatorios en el rango $(0,1)$. La ecuación 2a implica que cada partícula debe registrar su ubicación actual $x_{i d}$, su velocidad $v_{i d}$ y los vectores $p_{i d}$ y $p_{g d}$ representan los mejores valores de aptitud encontrados. Estos valores de aptitud se actualizan en cada generación, de acuerdo a:

$$
p_{i}(t+1)= \begin{cases}p_{i}(t) & f\left(x_{i}(t+1)\right) \leq f\left(x_{i}(t)\right) \\ x_{i}(t+1) & f\left(x_{i}(t+1)\right)>f\left(x_{i}(t)\right)\end{cases}
$$

donde el símbolo $f$ representa la función de fitness; $p_{i}(t)$ representa los mejores valores de fitness y las coordenadas donde fueron encontrados; y $t$ una nueva iteración en la generación.

\section{Aplicación de PSO para Clustering}

Es posible ver al problema de clustering como un problema de optimización que localiza los centroides óptimos de los clusters en lugar de encontrar la partición óptima. A diferencia de la búsqueda localizada del algoritmo de $K$-means, el algoritmo de clustering con PSO realiza una búsqueda global del espacio de búsqueda. Con el fin de combinar ambas características se utiliza un algoritmo de clustering híbrido PSO+K-means. En el algoritmo de PSO+K-means se combina la habilidad de la búsqueda globalizada del algoritmo de PSO con la rápida convergencia del algoritmo de $K$-means. Con la búsqueda global se garantiza que cada partícula busque en forma amplia cubriendo todo el espacio del problema y con la búsqueda local se trata de que todas las partículas converjan al óptimo cuando una partícula se acerca a la vecindad de la solución óptima.

En este contexto, una partícula representa los $k$ centroides de los clusters. Es decir que cada partícula $x_{i}$ se construye de la siguiente manera:

$$
x_{i}=\left(c_{1}, \ldots, c_{j}, \ldots, c_{K}\right)
$$

donde $c_{j}$ representa el $j$-ésimo centroide de la $i$-ésima partícula en el cluster $C_{j}$. El fitness de cada partícula se calcula en ambos algoritmos (PSO y PSO+K-means) de la siguiente forma:

$$
f(x)=\frac{\sum_{j=1}^{K}\left[\sum_{z \in C_{j}} d\left(z, c_{j}\right)\right]}{n}
$$

donde $d$ es la distancia Euclideana y $n$ es la cantidad de elementos del conjunto de datos, $z$ representa un elemento del conjunto de datos y $K$ es la cantidad de clusters. 


\subsection{PSO+K-means}

A continuación se describe en detalle el procedimiento de PSO+K-means. Para el caso del algoritmo PSO simple para clustering, el algoritmo es el mismo, excepto que éste no realiza los pasos $(d)$ y $(e)$ correspondientes a la hibridación con el K-means.

1. Inicializa cada partícula con $k$ centroides seleccionados aleatoriamente dentro del conjunto de datos.

2. Para $t=1$ a $t_{\max }$ hacer

a) Para cada partícula $x_{i}$ hacer

- Para cada vector $z$ del conjunto de datos

1) Calcular la distancia Euclideana $d\left(z, c_{j}\right)$ a todos los centroides $C_{j}$

2) Asigna $z$ a su cluster $C_{j}$ más cercano tal que la distancia a ese cluster sea la mínima.

3) Calcula el fitness usando la ecuación 5.

b) Actualiza la mejor posición global y la mejor posición local.

c) Actualiza la velocidad y posición de la partícula usando la ecuación 2a y 2b, respectivamente.

d) La mejor partícula encontrada es el vector de centroides para el proceso de $K$-means.

1) asigna cada elemento a su cluster más cercano.

2) recalcula cada centroide $c_{j}=\frac{1}{\left|C_{j}\right|} \sum_{\forall z \in C_{j}}$; donde $\left|C_{j}\right|$ corresponde al número de elementos en el cluster $C_{j}$.

3) Repetir los pasos $2 d 1$ y $2 d 2$ hasta alcanzar la condición de parada.

e) Reemplazar la mejor partícula global con los centroides obtenidos por $K$-means.

3. $t_{\max }$ es el número máximo de iteraciones. Finalizar cuando se alcance $t_{\max }$.

\section{Medidas de Calidad de Clustering}

A los efectos de establecer la calidad de los clusters encontrados por los distintos algoritmos estudiados, hemos considerado una serie de medidas no-supervisadas. Las medidas no-supervisadas de validez de un cluster pueden dividirse en dos clases. Medida de cohesión de los clusters (compacto, apretado) que determina cuán cercanos están los objetos dentro del cluster y la separación (aislamiento) que determina lo distinto y bien separado que está un cluster con respecto a los otros. Estas medidas a menudo son llamadas índices internos debido a que usan sólo información presente en el conjunto de datos. La calidad de los clusters obtenidos por cada algoritmo se analizó según los siguientes índices internos:

- Cuantización del error:

$$
\text { Error }-C=\frac{\sum_{i=1}^{K}\left(\sum_{z \in C_{i}} d\left(c_{i}, z\right) /\left|C_{i}\right|\right)}{K}
$$

- Distancia Intra-Cluster (cohesión). Distancia entre todos los elementos de un cluster, el objetivo es minimizar esta distancia:

$$
\text { Intra }-C=\frac{\sum_{i=1}^{K}\left(\sum_{z, t \in C_{i}} d(z, t) /\left|C_{i}\right|\right)}{K}
$$

- Distancia Inter-Cluster (separación). Distancia entre los centroides de los clusters, donde el objetivo es maximizar la distancia entre ellos:

$$
\text { Inter }-C=\frac{\sum_{i=1}^{K-1} \sum_{j=i+1}^{K} d\left(c_{i}, c_{j}\right)}{\sum_{i=1}^{K-1} i}
$$




\section{Experimentos y Resultados}

\subsection{Conjunto de Datos}

Los algoritmos se probaron con tres tipos de conjuntos de datos generados artificialmente y con tres conjuntos de datos provistos por Donald Bren School of Information and Computer Sciences (http://mlean.ics.uci.edu/MLSummary.html). Los datos artificiales tienen las siguientes características:

- Se utilizó una distribución Normal y se generaron números aleatorios a partir del método de Box-Müller. Se generaron instancias con elementos de 2, 3, 10, 50 y 100 dimensiones, con el fin de poder trasladar estos datos a problemas reales con dimensiones grandes que representen distintos atributos de un elemento. Además de trabajar con tamaños de conjuntos de datos de 600, 800 y 1000 elementos. Cada conjunto de datos estaba formado por cuatro clusters donde los elementos se distribuyeron de forma tal de obtener tres tipos diferentes de clusters:

- Clusters de igual cantidad de elementos, bien globulares y separados (figura 1(a)).

- Clusters de igual cantidad de elementos, con forma elíptica y entremezclados (figura 1(b)).

- Clusters de diferente cantidad de elementos, bien globulares y separados (figura 1 (c)).

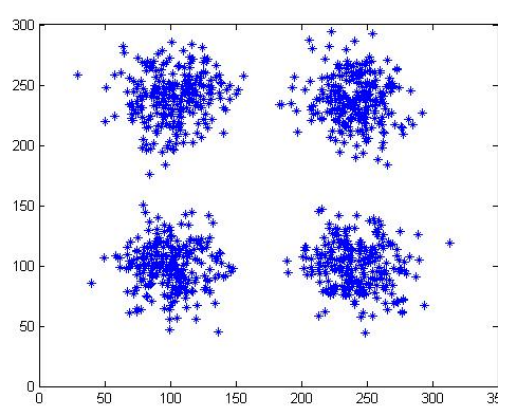

(a)

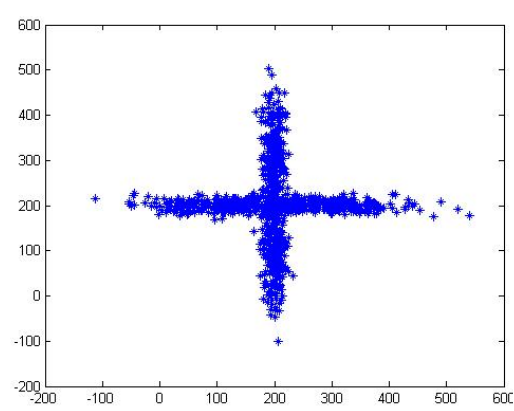

(b)

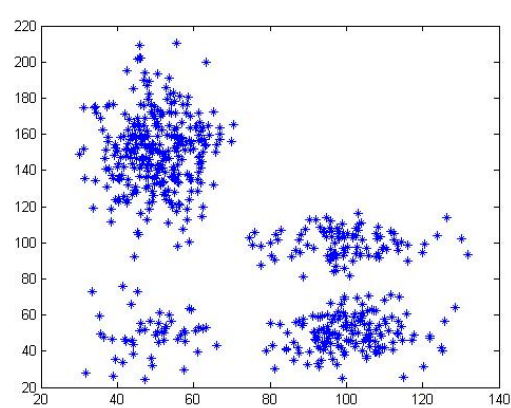

(c)

Figura 1: Conjunto de tres tipos de datos artificiales

La Figura 1 muestra tres conjuntos de datos artificiales de dimensión 2, con 1000 elementos cada uno (Instancias denominadas I26Ba2-1000, I41Bb2-1000, I56Bc2-1000; el nombre de cada instancia se forma de la siguiente manera por ejemplo I24Ba50-800 representa lo siguiente: $I 24$ identificador de la instancia $B a$ se refiere a la distribución de los elementos en el conjunto de datos tipo 1(a), 50 es la dimensión y 800 es la cantidad de puntos en el conjunto de datos). Con las características anteriormente descritas se generaron 45 instancias que para la generación de las gráficas que se muestran en la siguiente sección se agruparon de la siguiente manera:

- Grupo (a) está formado por 15 instancias con los datos distribuidos con la forma de la figura 1 (a) donde cinco instancias tienen dimensiones 2, 3, 10, 50 y 100 con 600 elementos del conjunto de datos cada una, otras cinco instancias tienen dimensiones $2,3,10,50$ y 100 con 800 elementos del conjunto de datos cada una y las cinco últimas instancias tienen dimensiones $2,3,10,50$ y 100 con 1000 elementos del conjunto de datos cada una.

- Grupo (b) las 15 instancias están agrupadas de manera similar al grupo (a) pero en este grupo los datos están distribuidos como se muestra en la figura 1(b).

- Grupo (c) las 15 instancias están agrupadas de manera similar al grupo (a) pero en este grupo los datos están distribuidos como se muestra en la figura 1(c).

El conjunto de datos reales está formado por las siguientes Bases de datos:

- "Iris plants": es una base de datos con 4 atributos numéricos, 3 clases y 150 elementos.

- "Glass identification": esta base de datos contiene 6 tipos de vidrio definidos en términos de su contenido de óxido (es decir $\mathrm{Na}, \mathrm{Fe}, \mathrm{K}$, etc).

- "Teaching assistant evaluation"(TAE): esta base de datos contiene 151 elementos, 6 atributos, 3 clases. Los datos corresponden a la performance de la enseñanza de tres semestres regulares y dos semestres de verano de 151 asistentes de enseñanza en la Universidad de Wisconsin - Madison. 


\subsection{Configuración de Algoritmos y Resultados}

Para todos los algoritmos se realizaron 30 corridas independientes. En PSO y PSO+K-means se utilizó para espacios de problema de dimensiones pequeñas $(<10)$ un cúmulo de partículas de 15 y para dimensiones mayores $(\geq 10)$ el cúmulo utilizado fue de 50 partículas. Los valores de las contantes $c_{1}$ y $c_{2}$ se fijaron en 1.49 y $w=0,72$.

A continuación se comparan los resultados de los algoritmos de PSO, K-means y PSO+K-means, de 45 instancias artificiales se seleccionaron tres instancias de cada grupo y las tres instancias que pertenecen al conjunto de datos reales.

En la tablas 1, 2 y 3 se muestran los resultados obtenidos en cada algoritmo de los promedios de 30 corridas independientes para las instancias analizadas, donde la columna: "Instancia" corresponde a la instancia elegida, "C-Error" muestra la cuantización del error, "Intra-C" corresponde a la distancia Intra-Cluster, "Inter-C" es la distancia Inter-

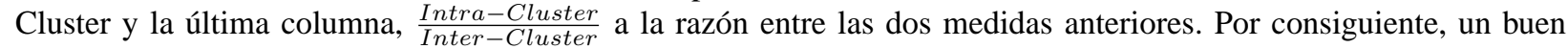
clustering debería ser aquel que contenga cada uno de los clusters individuales homogéneos y entre todos los clusters tan heterogéneos como sea posible. Donde un valor pequeño de $\frac{I n t r a-C l u s t e r}{\text { Inter-Cluster }}$ representa homogeneidad dentro de los clusters y heterogeneidad entre los clusters.

Se puede observar que en todos los casos seleccionados del conjunto de datos artificiales y para la instancia Iris-Plants el algoritmo PSO $+K$-means obtiene mejores resultados para cada una de las medidas de calidad analizadas. En estas instancias se obtiene una menor cuantización del error, una menor distancia Inter-Cluster (más separación) y una distancia Intra-Cluster menor (más agrupamiento en los elementos) con lo cual significa que este algoritmo híbrido obtiene clusters más cohesionados y separados entre ellos.

Tabla 1: Resultados obtenidos con PSO

\begin{tabular}{|l|c|c|c|c|}
\hline Instancia & C-Error & Intra-C & Inter-C & $\frac{\text { Intra }-C}{\text { Inter }-C}$ \\
\hline I22Ba3-800 & 36,96 & 46,31 & 161,74 & 0,2864 \\
I23Ba10-800 & 81,04 & 89,65 & 282,26 & 0,3176 \\
I24Ba50-800 & 153,91 & 199,38 & 654,94 & 0,3044 \\
I38Bb10-800 & 166,84 & 213,90 & 316,29 & 0,6763 \\
I39Bb50-800 & 407,95 & 481,96 & 741,84 & 0,6497 \\
I43Bb10-1000 & 176,35 & 208,69 & 304,80 & 0,6847 \\
I58Bc10-1000 & 50,05 & 55,16 & 139,46 & 0,3955 \\
I59Bc50-1000 & 118,49 & 119,64 & 311,24 & 0,3844 \\
I60Bc100-1000 & 169,12 & 168,68 & 446,02 & 0,3782 \\
Iris-Plants & 0,72 & 0,93 & 3,16 & 0,2931 \\
Glass & 1,46 & 1,97 & 3,65 & 0,5390 \\
TAE & 11,21 & 14,22 & 22,19 & 0,6409 \\
\hline
\end{tabular}

Tabla 2: Resultados obtenidos con $K$-means

\begin{tabular}{|l|c|c|c|c|}
\hline Instancia & C-Error & Intra-C & Inter-C & $\frac{\text { Intra-C }}{\text { Inter-C }}$ \\
\hline I22Ba3-800 & 31,94 & 45,25 & 159,85 & 0,2831 \\
I23Ba10-800 & 67,40 & 93,85 & 289,76 & 0,3239 \\
I24Ba50-800 & 161,31 & 222,10 & 623,29 & 0,3563 \\
I38Bb10-800 & 156,46 & 220,65 & 334,98 & 0,6587 \\
I39Bb50-800 & 369,07 & 518,64 & 713,08 & 0,7273 \\
I43Bb10-1000 & 151,10 & 213,17 & 341,13 & 0,6249 \\
I58Bc10-1000 & 38,98 & 54,67 & 140,82 & 0,3883 \\
I59Bc50-1000 & 95,78 & 133,88 & 314,64 & 0,4255 \\
I60Bc100-1000 & 138,56 & 194,35 & 426,41 & 0,4558 \\
Iris-Plants & 0,65 & 0,92 & 3,27 & 0,2825 \\
Glass & 1,58 & 2,29 & 4,34 & 0,5286 \\
TAE & 10,15 & 13,96 & 20,98 & 0,6657 \\
\hline
\end{tabular}

Tabla 3: Resultados obtenidos con PSO+Kmeans

\begin{tabular}{|l|c|c|c|c|}
\hline Instancia & C-Error & Intra-C & Inter-C & $\frac{\text { Intra-C }}{\text { Inter }-C}$ \\
\hline I22Ba3-800 & 31,67 & 44,90 & 160,95 & 0,2790 \\
I23Ba10-800 & 62,33 & 88,32 & 298,37 & 0,2960 \\
I24Ba50-800 & 151,19 & 199,38 & 659,56 & 0,3023 \\
I38Bb10-800 & 151,27 & 214,48 & 356,83 & 0,6011 \\
I39Bb50-800 & 361,26 & 481,96 & 788,53 & 0,6112 \\
I43Bb10-1000 & 146,40 & 192,03 & 445,42 & 0,4311 \\
I58Bc10-1000 & 36,64 & 51,43 & 147,21 & 0,3493 \\
I59Bc50-1000 & 90,11 & 115,02 & 334,93 & 0,3434 \\
I60Bc100-1000 & 136,52 & 161,93 & 469,57 & 0,3449 \\
Iris-Plants & 0,64 & 0,92 & 3,28 & 0,2816 \\
Glass & 1,44 & 1,96 & 3,84 & 0,5117 \\
TAE & 9,83 & 13,71 & 20,73 & 0,6610 \\
\hline
\end{tabular}

Adicionalmente, en la figura 2 se muestran los respectivos valores de $\frac{\text { Intra-Cluster }}{\text { Inter-Cluster }}$ de los tres algoritmos para el grupo de 15 instancias denominado grupo (a). Se puede observar que con PSO+K-means se obtienen mejores

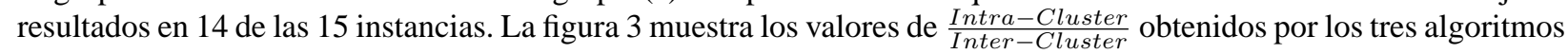
para las instancias del grupo (b). Se puede observar que con PSO+K-means se obtienen mejores resultados en 9 de las 15 instancias. Por su parte, en la figura 4 se muestran los valores $\frac{\text { Intra-Cluster }}{\text { Inter-Cluster }}$ de los tres algoritmos para las instancias del grupo (c). En este caso se puede observar que con esta distribución de los datos con el algoritmo PSO+K-means 
se obtienen mejores resultados en todas y cada una de las 15 instancias. Por último, para el conjunto de datos reales figura 5 se obtienen mejores resultados en dos de las 3 instancias.

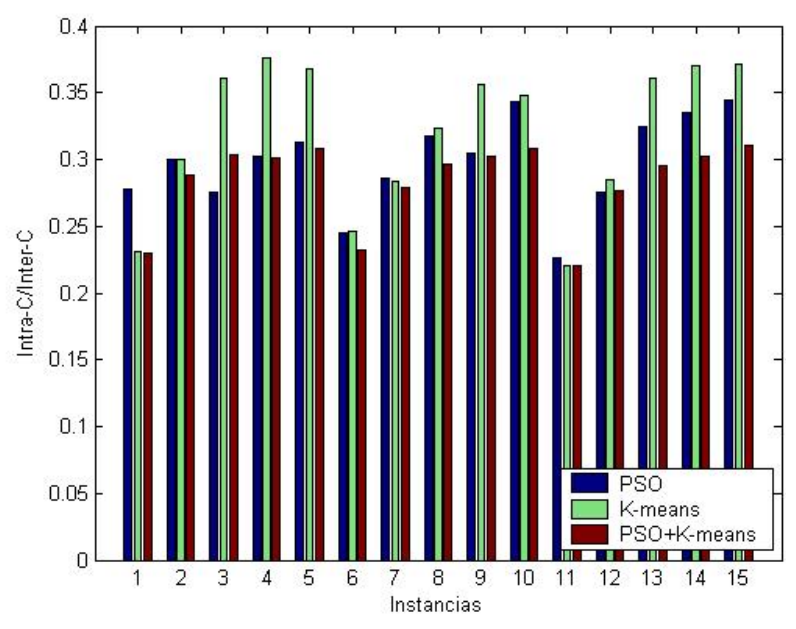

Figura 2: Valores $\frac{\text { Intra-Cluster }}{\text { Inter-Cluster }}$ para el grupo a

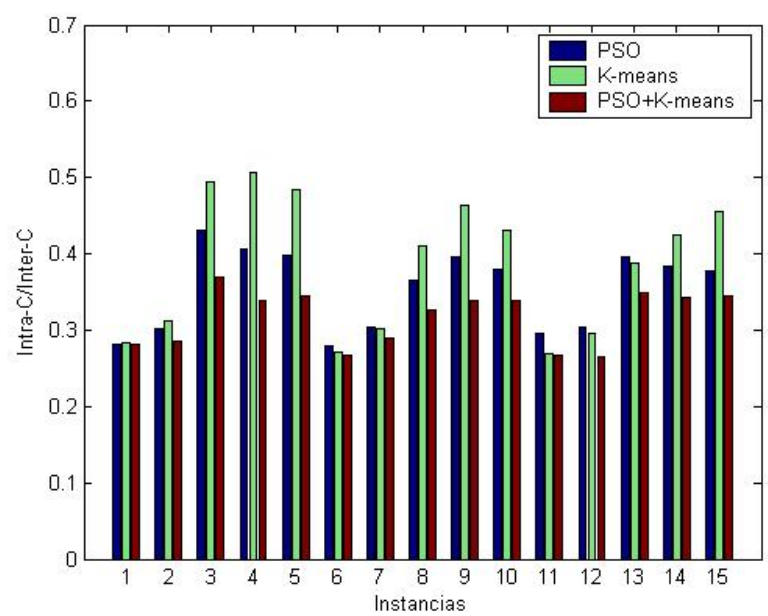

Figura 4: Valores $\frac{\text { Intra-Cluster }}{\text { Inter-Cluster }}$ para el grupo c

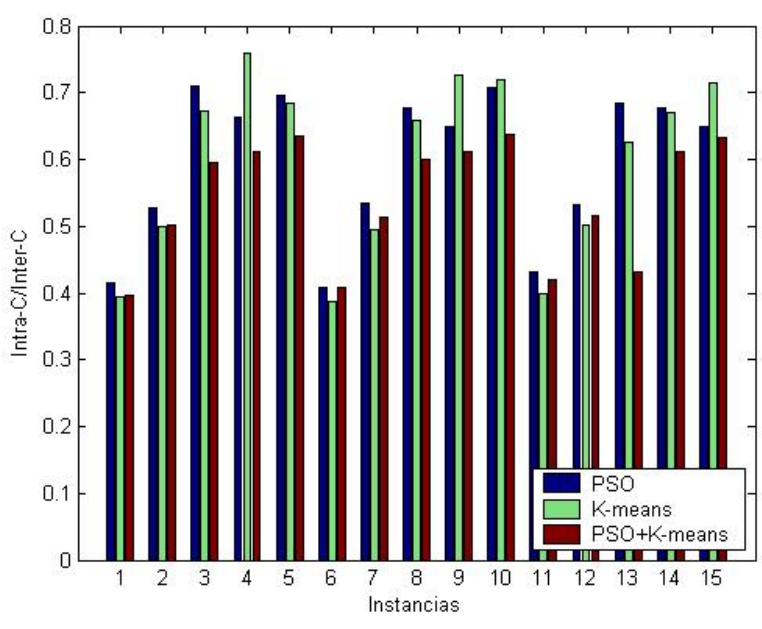

Figura 3: Valores $\frac{\text { Intra-Cluster }}{\text { Inter-Cluster }}$ para el grupo b

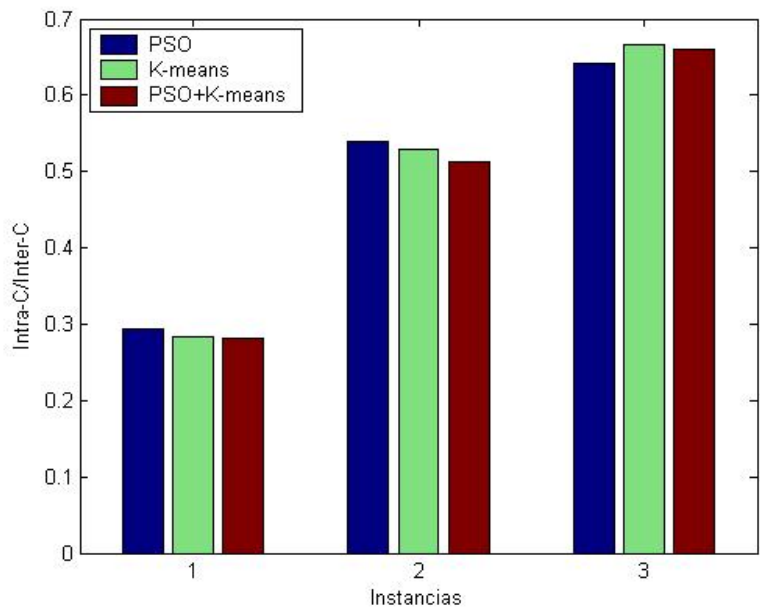

Figura 5: Valores $\frac{\text { Intra-Cluster }}{\text { Inter-Cluster }}$ para el conjunto de datos reales, donde $1=$ Iris, $2=$ Glass y $3=$ TAE

\section{Conclusiones}

PSO es una técnica de optimización que realiza una búsqueda globalizada de las soluciones y puede aplicarse a problemas de clustering. K-means es un algoritmo simple, directo y muy utilizado en clustering pero puede quedar atrapado en óptimos locales. En este trabajo se analizaron medidas de calidad de los clusters obtenidos por tres algoritmos PSO, K-means y PSO+K-means, este último combina la habilidad de la búsqueda globalizada de PSO con la búsqueda local de $K$-means. Se mostró que PSO+K-means logró encontrar en la mayoría de los casos analizados mejores resultados comparados por los obtenidos por $K$-means y PSO de manera individual con respecto a la cuantización del error, y las distancias inter-cluster e intra-cluster. Es decir que este algoritmo híbrido en general logró encontrar clusters más cohesionados y separados. En futuros trabajos se analizará la calidad y el número clusters obtenidos por este algoritmo híbrido así como también el efecto de usar diferentes parámetros en la performance de la convergencia de PSO+K-means.

\section{Agradecimientos}

Los autores agradecen a la Universidad Nacional de la Patagonia Austral por su apoyo al grupo de investigación y además, la cooperación de los integrantes del proyecto que continuamente proveen de nuevas ideas y críticas con- 
structivas. El cuarto autor agradece además, el constante apoyo brindado por la Universidad Nacional de San Luis y la ANPYCIT que financian sus actuales investigaciones.

\section{Referencias}

[1] Jain A.K., Murty M. N., and Flynn P. J. Data clustering: A review. ACM Computing Survey, 31(3):264-323, 1999.

[2] Jain A.K. and Dubes R.C. Algorithms for Clustering Data. Englewood Cliffs, N.J.:Prentice Hall, 1988.

[3] Chui-Yu Chui, Yi-Feng Chen, I-Ting Kou, and He Chun Ku. An intelligent market segmentation system using k-means and particle swarm optimization. Expert Systems with Applications, 2008.

[4] Kennedy J. and Eberhart R. Swarm Intelligence. Morgan Kaufmann, San Francisco, California, 2001.

[5] MacQueen J. Some methods for classification and analysis of multivariate observations. In Proceedings of the 5th Berkeley Symp. Math. Statist, Prob, pages 281-297, 1968.

[6] Omran M., Salman A., and Engelbrecht A.P. Image classification using particle swarm optimization. In Conference on Simulated Evolution and Learning, Computational intelligence for the E-age, 2002.

[7] Tou J. T. and Gonzalez R. C. Pattern recognition principles. Addison-Wesley, 1974.

[8] Fayyad U., Piatetsky-Shapiro G., and Smith P. From data mining to knowledge discovery in database. In American Association for Artificial Intelligence, pages 37-54, 1996.

[9] Backer U.E. Computer-assisted reasoning in cluster analysis. Prentice-Hall, 1995.

[10] Huang T. W. Application of clustering analysis for reducing smt setup time- a case study on avantech company. Master's thesis, Department of National Taipei University of Technology, 2006.

[11] Selim S. Z. and Ismail M.A. K-means type algorithms: A generalized convergence theorem and characterization of local optimality. IEEE Trans. Pattern Anal. Mach. Intell., (6):81-87, 1984. 